\title{
Discussion forum rubrics: using rubrics to enhance and increase project management students' participation in online discussions
}

Margaret R. Lee, LEE Curriculum Consultants, USA

Deborah S. Carstens, Florida Institute of Technology, USA

Linda C. Malone, University of Central Florida, USA

\begin{abstract}
This paper examines the impact of using a discussion forum grading rubric to encourage and enhance discussions (posts) in an online introduction to project management course.

The study consists of 53 Master of Business Administration (MBA) students enrolled in three sections of the course from October 2010 to May 2011. A control group of 20 students was compared to two experimental groups of 16 and 17 students respectively. The two experimental groups were given the specific grading rubric and instructions designed to encourage online conversation; the control group was not.

The results indicate that there was a statistical difference in average postings per student between the two experimental groups and the control group but no statistical difference between the two experimental groups. The results suggest that the use of a rubric specifically designed to engage the student and expand the student's learning experience can increase online classroom participation and, as a consequence, enhance the educational experience for the online project management student and strengthen the university's MBA program.
\end{abstract}

\section{Keywords}

discussion forums, education, online, participation, project management, rubric

\section{Background}

It can be difficult to move online students to a formal rubric-based grading system for discussion forums, but the benefits are effective and measurable. Therefore, the purpose of the research was to examine the impact of using a discussion forum rubric to enhance discussion posts submitted by students in the virtual project management classroom. The analysis involved evaluating the first section of the virtual project management classroom in which the protocols and evaluation criteria were minimal or loosely defined, as well as the remaining sections of the course in which the opposite was true. The evaluation involved how the addition of facilitator guidelines influenced meaningful discourse, how the posting of these guidelines influenced meaningful discourse, and how the addition of an evaluation rubric influenced meaningful discourse.

The students often go kicking and screaming into the formal grading process. The following quote from a course evaluation demonstrates this:

\section{ABSOLUTELY HATED THE DISCUSSION QUESTION REQUIREMENT to} search for and provide a response with references from the text and an outside source PLUS [c]ite in APA format. That said it was probably the most beneficial part of the course. BUT I STILL HATE IT. 
In teaching online project management classes, the grading rubric adds yet another dimension, as demonstrated by this student's insightful comments to the other learners in the class:

As a PM I think that the harsh discussion requirements are right in tune with reality and that the difficulties are a learning opportunity in and of themselves. When you are managing a project and disagree with the customer on a requirement (that you agreed upon by signing), you are rarely going to see the results you expect. Especially if nothing has been said right up until the time it became difficult for you to execute ... Let's look at this course as if we were project managers.

- Project initiation stage - We sign up for the course and the instructor agrees to teach the course.

- Project planning and design stage - We prepare for the course and the instructor outlines the syllabus.

- Project execution and construction stage - This would be week one. You can still get a return on your investment if you decide the course is not for you. You have a chance to review the course materials (requirements) and to question them. By saying nothing, signing the statement of understanding and remaining in the course you have agreed to the project terms.

- Project monitoring and controlling systems - We continue with the course work.

- Project completion - Final Exam and hopefully, the taking from this course a wealth of knowledge that will prove useful in your career.

The research outcome is important as inclusion of effective grading rubrics for discussion forums in online project management courses represents a movement toward higher standards in the classroom, increased satisfaction and learning for the students, and improved quality and quantity of online discussions. These improvements should, as a result, enhance and strengthen the university's online project management program.

\section{Introduction}

The explosion of online learning presents opportunities and challenges for universities to maintain quality in education while increasing satisfaction among learners. As business schools compete to capture market share in this competitive arena, they must also maintain the highest standards of excellence and the best learning environment for their learners (Blount 2011: 390-404). To do this, many institutions have developed online learning courses for the convenience of their students. The purpose of this research was to examine the impact of using a discussion forum rubric to enhance discussion posts submitted by students in the virtual project management classroom.

This need to develop high-quality online learning experiences requires instructional methods that ensure online discussion forums to provide a sense of community, interaction and social presence and thus foster learning and learner satisfaction. Student satisfaction surveys for Masters of Business Administration (MBA) degrees reveal that traditional online discussion forum questions do not encourage critical thinking compared with lively in-class interactions (Strang 2011: 80-92). The ability and necessity to develop a sense of community with virtual learners in the online environment is dependent upon the instructors and other learners sharing insights and knowledge (Menkhoff et al 2011: 132-151). Research shows that students who feel a sense of community in the online environment are more likely to continue in an online program than students who feel alone or alienated (Rovai 2003: 89-107). This, in 
turn, leads to an increase in high-quality e-learning and course completion rates and to the success of the academic program.

Current research suggests that an interactive, collaborative learning model is appropriate for online MBA programs due to the prior business experience of the learners and the convenient, asynchronous learning environment (Arbaugh \& Rau 2007). Menkhoff et al's (2011: 132-151) study indicates that social presence is positively related to the students' desire to learn. Owston (1997) states that, in his experience teaching graduate-level courses, online discussions are often more thoughtful and reflective, with quiet or shy students more eager to interact than in the traditional classroom. Students have also reflected on the online learning process as a successful medium. Langan (1997: 129) writes of her online experiences, stating: "The online environment is much richer in resources and discussions founded in sound research and critical thinking than I have found in a local classroom environment". Berge and Collins (1995) concur, stating that online learning encourages an active environment of social learning in which learners can explore their own interests, interact with peers beyond their own local network, and communicate with peers in a learning atmosphere.

The importance of student-to-student interaction is essential to the establishment of the online learning community. Dawson et al (2006: 127-139) posit that increased student interaction is an indicator of the development of the community, and quantitative measures of student-tostudent interactions occurring online in discussion forums are useful in benchmarking and analysing online classes. In a quasi-experiment comparing two instructional approaches for an online MBA business strategy class, Strang (2011: 80-92) explored how discussion questions might be more effective in online MBA classes. He hypothesised and proved that more online experiential knowledge interactions had a positive effect on the students' grades and the number of online interactions. Menkhoff et al (2011: 132-151) hypothesised that the frequency of online interaction stimulated the desire to learn, and that student-to-student interaction stimulated interest in the topic. Their study of 138 online undergraduate business management students indicated a positive relationship between the e-learning experience and the frequency of online interaction. Arbaugh (2005: 135-149), in his study of 44 MBA online classes between 1998 to 2001, hypothesised that interaction between class participants would be positively associated with perceived learning and satisfaction with the MBA classes. His results strongly supported these hypotheses and indicated that encouraging participation and collaboration may enhance online learning. Swan (2001: 306-331) looked at 1,108 online students in 73 SUNY Learning Network courses in the spring semester of 1999. She found that students with higher levels of activity in the courses and high levels of student-to-student interaction reported higher levels of satisfaction and higher levels of learning from the classes. That is, the "greater the percentage of the course grade that was based on discussion, the more satisfied the students were, the more they thought they learned from the course, and the more interaction they thought they had with the instructor and with their peers" (Swan 2001:306-331).

The challenge facing the online instructor is in involving learners in the online discussion forums and engaging the students in quality discussions (Gilbert \& Dabbagh 2005: 5-18). There appears to be an absence of a comprehensive, theory-driven metric that can assess quality and effectiveness of the discussion forum in online classrooms (Kay 2006: 761-783). The amount of quality interaction between learners in online classes is a well-established indicator of virtual course effectiveness and it also positively predicts student learning and satisfaction (Arbaugh \& Rau 2007). With a practice-based subject like project management, 
the design, development and facilitation of discussion forums becomes a critical component to the success of the educator's curriculum. The asynchronous environment allows for careful reflection and thoughtfulness in posts and an equal playing field for all students (Fauske \& Wade 2004: 137-153). Through robust online discussions, virtual learners collaborate to share practical ideas, best practices, tools and techniques, as well as expand their learning experience and, therefore, increase their satisfaction with the curriculum. A student's use of terms in a post or increase in verbal activity alone may not indicate an increase in learning (Fauske \& Wade 2004: 137-153); therefore, a consistent, fair and equitable means of assessing the interaction and quality of the posts, such as the use of a rubric, is necessary. Current research literature rarely discusses the kind and degree of structure that can be effective in developing quality student-to-student interactions and discussions.

A rubric is a scoring tool for a piece of work and can be a helpful tool for both the learner and the instructor (Goodrich 1996: 14-17), providing a guide to evaluate the quality of students' constructed responses using three features - evaluative criteria, quality definitions and a scoring strategy (Popham 1997: 72-75). In his study of 262 graduate-level students in 18 online courses, Rovai (2003: 89-107) found that grading strategies influence online discussions and that these discussions relate directly to the students' sense of community. The rubric criteria in his study included the number of posts each week, the number of accesses to the discussion board weekly, and the content of the posts. His results showed that courses in which discussions were graded had significantly more discussions per student per week and higher levels of a sense of community, and also students who felt more satisfied with their education and the online experience. Benefits of using rubrics include: (a) efficiency in grading assignments, (b) validity and accuracy of grading, (c) similarity of grading tools used in the workplace, and (d) clarity of expectations for the student (Czaplewski 2009: 29-36). In addition, rubrics can clarify expectations for the instructor, help students focus on the work and provide unbiased feedback without excessive instructor grading (Goodrich Andrade 2005: 27-30). The result of using rubrics is often improvement in the quality of work and the learning experience for the student (Goodrich 1996: 14-17).

Several problems are cited in the literature regarding the design and use of rubrics. Among those listed by Czaplewski (2009: 29-36) are: (a) rubrics that are too complicated or detailed, (b) the learners' inability to agree with the grade, and (c) rubrics are often misused as a substitute for valuable teaching and mentoring opportunities. Other problems with rubrics can be that they are not entirely self-explanatory, they cannot replace good instruction, and students often are not good at self-assessment and, therefore, cannot properly use the rubric (Goodrich Andrade 2005: 27-30). Popham (1997: 72-75) suggests that flaws of rubrics include criteria that are not relevant or too general, missing the key attributes of the learning being assessed.

According to Czaplewski (2009: 29-36), a rubric must clearly outline the criteria and how each will be graded. Weltzer-Ward's (2011: 56-74) meta-analysis of online classroom discussions yielded several categories appropriate for development of the rubric for this introductory project management class. Some of her rubric categories included level of participation, interactivity, focus on or thoroughness of understanding the discussion prompt, professional tone, clarity and mechanics. The meta-analysis of online classroom discussions also suggests that the consensus of researchers is that the post (initial post responding to the discussion question and response posts to other learners) is the most appropriate and valid unit for analysis. Czaplewski (2009: 29-36) suggests comments can be written ahead by the 
professor and then copied and pasted into the feedback comments to increase the efficiency of the use of the rubric.

The discussion grading rubric and clear communication by the facilitator of the criteria in the rubric allows for a means of just assessment and evidence of the learner's interaction levels and quality of discussion posts. This study examines the impact of using such a discussion forum grading rubric to encourage and enhance discussions (posts) in the virtual project management classroom which, in turn, may assist educational facilities providing exceptional virtual project management classes and prompt the growth of the institution's online learning programs.

\section{Method}

\section{Comparable study}

This study is comparable to and attempts to repeat that of Gilbert and Dabbagh's (2005: 5-18) work that examined the impact of online discussion protocols and evaluation rubrics on meaningful discourse in the online classroom. Their study involved 87 participants from four sections of a graduate course in instructional technology foundations and learning theory from spring 1999 to spring 2001. In the first section, protocols and evaluation criteria were minimal or loosely defined. In the remaining sections, the opposite was true. They reviewed how the addition of facilitator guidelines influenced meaningful discourse, how the posting of these guidelines influenced meaningful discourse, and how the addition of an evaluation rubric influenced meaningful discourse. Their analysis found that providing guidelines and using a rubric improved not only the facilitation and evaluation of the online discussions, but also increased the number and quality of the students' posts, improving them by students' abilities to: (a) promote and relate new knowledge to prior knowledge and experience, (b) interpret content, (c) analyse, synthesise and evaluate other students' posts, and (d) make inferences. Therefore, Gilbert and Dabbagh's (2005) study suggested that using facilitator guidelines, posting protocols explaining discussion board expectations relating to the quantity and quality of posts, and following evaluation criteria improved students' posts.

The current study is similar in that facilitator guidelines, posting protocols and evaluation criteria were also used although this study used different guidelines, protocols and evaluation criteria. The current study is set in a project management course whereas Gilbert and Dabbagh's (2005) study was set in an instructional technology foundations and learning theory course. However, the largest difference is that Gilbert and Dabbagh's (2005) study took place in a course in which students led the discussion board question and responses to other students, as this was a task in their syllabus as part of their grade. Students were required to form groups of two and each pair was assigned one week of leading the discussion board efforts. Thus, a different student team performed the facilitation of the discussion board each week. In the current study, the discussion board task was instructorled, being fully facilitated by the same instructor every week for all three sections.

\section{Participants}

This study consisted of 53 MBA students enrolled in three sections of an introduction to project management course from October 2010 to May 2011. The course is the first of four project management courses students are required to take in a series. MBA students are generally striving toward excellence in the workplace, and are willing to study to advance their careers and increase their opportunities (Li 2011: 201-220). They are commonly parttime students, looking not only to advance their careers, but also to understand theory and best practices (Elgort 2011: 97-108). 
A control group of 20 students was compared to two experimental groups of 16 and 17 students respectively. The participants of the study, a deliberate convenience non-random sample, were students in three separate classes. They were considered to be a random sample, as they were not assigned to any of the three classes specifically by the university, and all students must fulfil identical requirements to be admitted into the MBA program and the course.

\section{Rubric}

The rubric, which included instructions designed to encourage online conversation, was used in the two experimental groups but not in the control group. The rubric was simplified to include five columns and the grading for each column was either 0 or 5 , indicating that the learner either did not complete the task successfully (0) or completed the task successfully (5). This eliminated the learners' inability to agree with the grade. Issues with the rubric not being entirely self-explanatory were resolved by providing the rubric as an Excel spreadsheet. The learners are able to self-evaluate the quality of their posts and responses, and the totals will sum automatically to show the score by discussion question and by the total week score. This also mitigated the potential problem that students may not be able to properly use the rubric. The criteria used in the rubric were carefully constructed to be relevant to the key attributes of the learning being assessed. For example, sharing personal experiences and expertise is important for learning at the MBA level. The personal experiences that the learner shares in posts are considered under the Example/Experience section of the rubric (5 points) and also under the Insight/Depth section of the rubric (5 points), so they contribute twice the weight in grading the discussion questions.

To compensate for the possible lack of professor feedback to help the learner understand the requirements for posts or misuse of the rubric as a substitute for valuable teaching and mentoring opportunities, the rubric scores were presented along with comments to help the learners. Below are a few examples of the comments used for this project management class:

Great job this week! So much of what happens in project management is sharing ideas, best practices, tools and techniques. Thank you for making this happen in our discussion boards! In Week 4, go the extra mile and respond to more than just the minimum number (2) of learners for an outstanding week (-5 DQ\#2 Response).

I did not see that you used the book as a reference. It's important to use our book as a resource for the other learners' reference purposes and as a basic denominator for our class. Be sure to include our text(s) as a reference in your response(s) (-5 DQ \#1 Initial Post).

I noticed that you did not include outside references. It's important to use outside resource(s) for the other learners' reference purposes and to expand your own knowledge on the topic. I'd like to see you use some outside resources to round out your thinking on our topics (-5 DQ\#1 Response, -5 DQ\#2 Initial Post).

The rubric for this study scores the initial discussion post (in which the student answers a question posed by the facilitator) content on five levels: (1) if it is an insightful, thorough, and interesting analysis of material, whether or not the post includes new and useful information, and if the learner demonstrates understanding of the topic; (2) if it includes examples from the text; (3) if it includes examples of other academic resources; (4) 
completeness of response; and (5) if the learner shares personal examples and experiences on the subject. These categories encourage the students to consider additional perspectives and deep learning, versus just cursory discussion (Strang 2011: 80-92).

It also scores the content of the response posts written to other students on five levels. The first level (Insight/Depth) scores the response on the following: the response must be more than just agreement, it must answer another student's question(s) or lead that learner to a resource that will help answer his or her question(s); it must include in-depth evaluation or discussion, use personal stories, provide new resources, share examples or experiences that relate to the topic; and it must ask questions to encourage conversation and/or provide a contrasting view. It scores if the responder used a reference to the text and used additional academic references. Finally, the rubric scores the required number of responses to other learners, and increases the score (five points) when the learner responds to more than the minimum required number of learners. (See Appendix A for the rubric used in the two experimental groups.)

Detailed instructions for the two experimental groups for the grading process and the rubric were presented to the participants the first week of class (see Appendix B). Virtual students often self-advise, misunderstand instructions and, therefore, become less committed (Menkhoff, et al. 2011: 132-151), so clear instructions were provided for the rubric. Each student was required to respond to the post that included the instructions and the copy of the rubric as an Excel worksheet by posting a response that showed that he or she had read the instructions and rubric.

\section{Qualitative responses}

To substantiate the quantitative results, student evaluation comments were captured that related directly to the use of the rubric in the project management class and related comments from the control group were also captured. These comments can be found in Appendix C. The comments from the experimental groups suggest that although hard work was required to get a good grade for the discussion board posts, students felt that it forced them to learn more by being held to a higher standard and by having to interact more with others in the class. The control group evaluation comments consisted of only two comments but these addressed appreciation for feedback in the grading process in terms of how to improve the quality of a discussion board post and thus improve a student's grade. The comments also suggest the need for more interaction resulting in more learning in the control group as well, which was the group without the rubric.

\section{Results}

Statistical analysis

A statistical analysis was done comparing the average number of responses per student per discussion question among the three groups. Because the relative sample sizes were small and normality was not ensured, nonparametric statistics were used to compare the groups. First, a comparison was made on the average number of responses per question between the two experimental groups. The two groups were compared question by question (matching) over the entire semester of work. A matched pairs Wilcoxon Signed-Rank test was conducted because this test is used when comparing samples to assess whether their population mean ranks differ. No significant statistical difference was found between the two experimental groups $(p=0.272)$. Since no difference existed between the two experimental groups, they were combined and a comparison was made between the combined experimental group and the control group. A statistically significant difference $(p=0.000)$ 
was found using the Wilcoxon Rank-Sum Test because this test is used to assess whether one of two samples of independent observations tends to have larger values than the other.

Statistical analysis of the average of the four quiz grades for the students was also done using nonparametric statistics. First, the two experimental groups were compared using a Wilcoxon Rank-Sum Test, which revealed that there was no statistical difference between the two experimental groups at the $p=0.6013$ level. Because the two experimental groups were not statistically different, they were combined into one experimental group. When comparing the combined experimental groups to the control group, there was also no significant difference if one is using $\alpha=0.05$. However, there would be a difference at the $\alpha=0.10$ level.

\section{Instructor evaluations}

The instructor evaluations for the control group and experimental groups were reviewed. Histograms were created to display the student feedback collected at the end of each of the three sections, control group and two experimental groups. The histograms reflect the responses to those questions on the evaluation form that relate to any aspect of the discussion board questions.

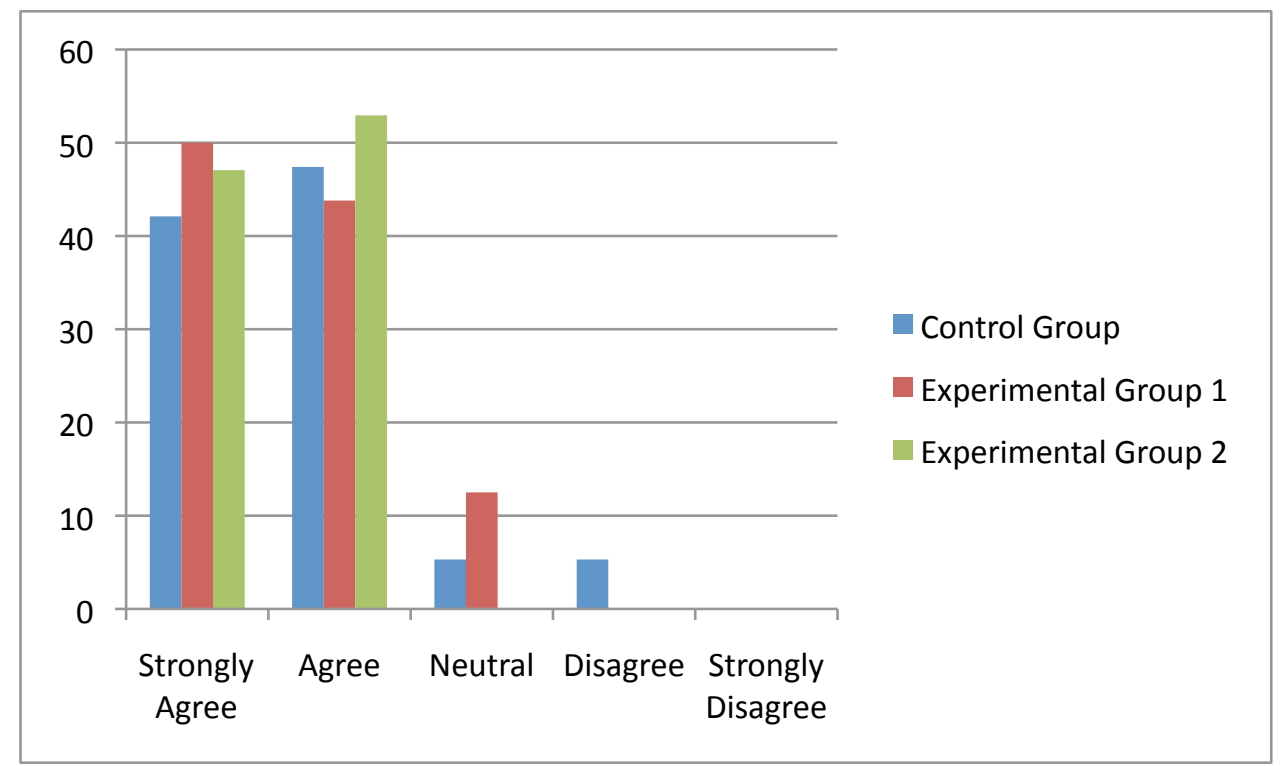

Figure 1. Student responses to the question "Hard work is required to get good grades in this course" 


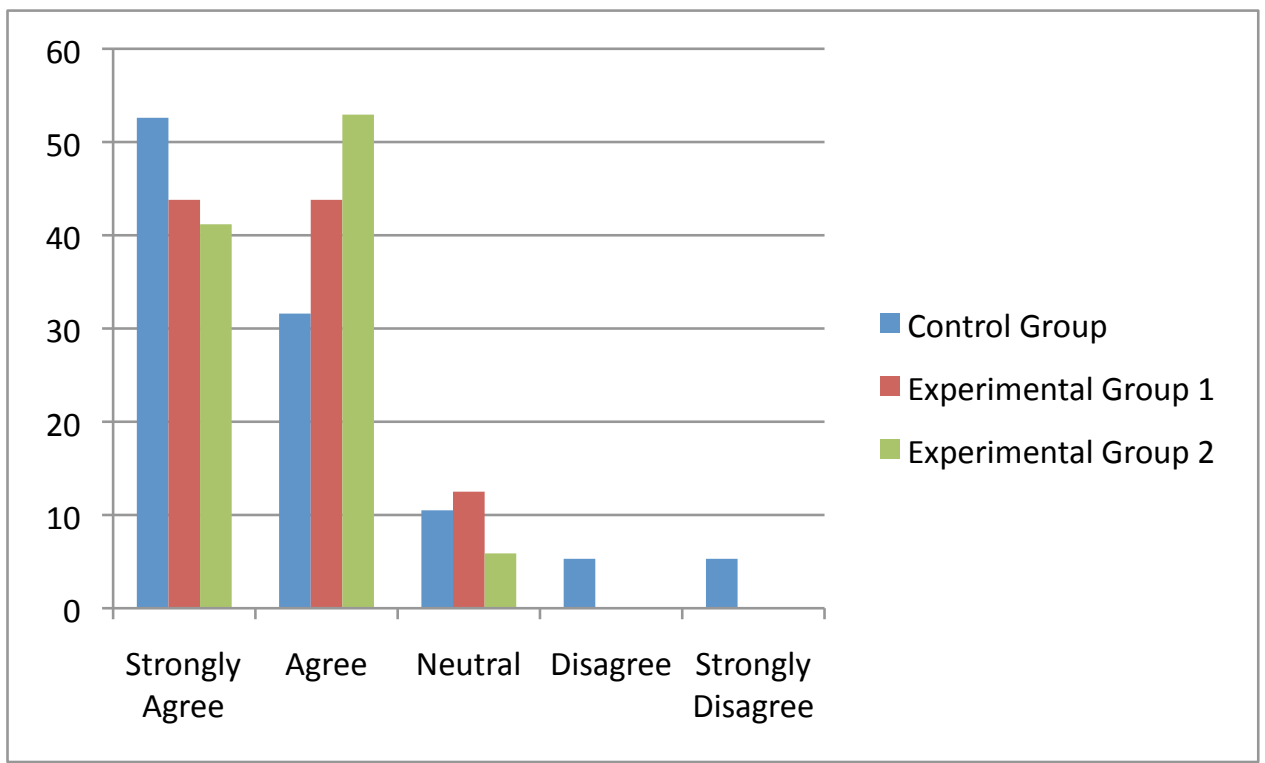

Figure 2. Student responses to the question

"I found the course intellectually stimulating"

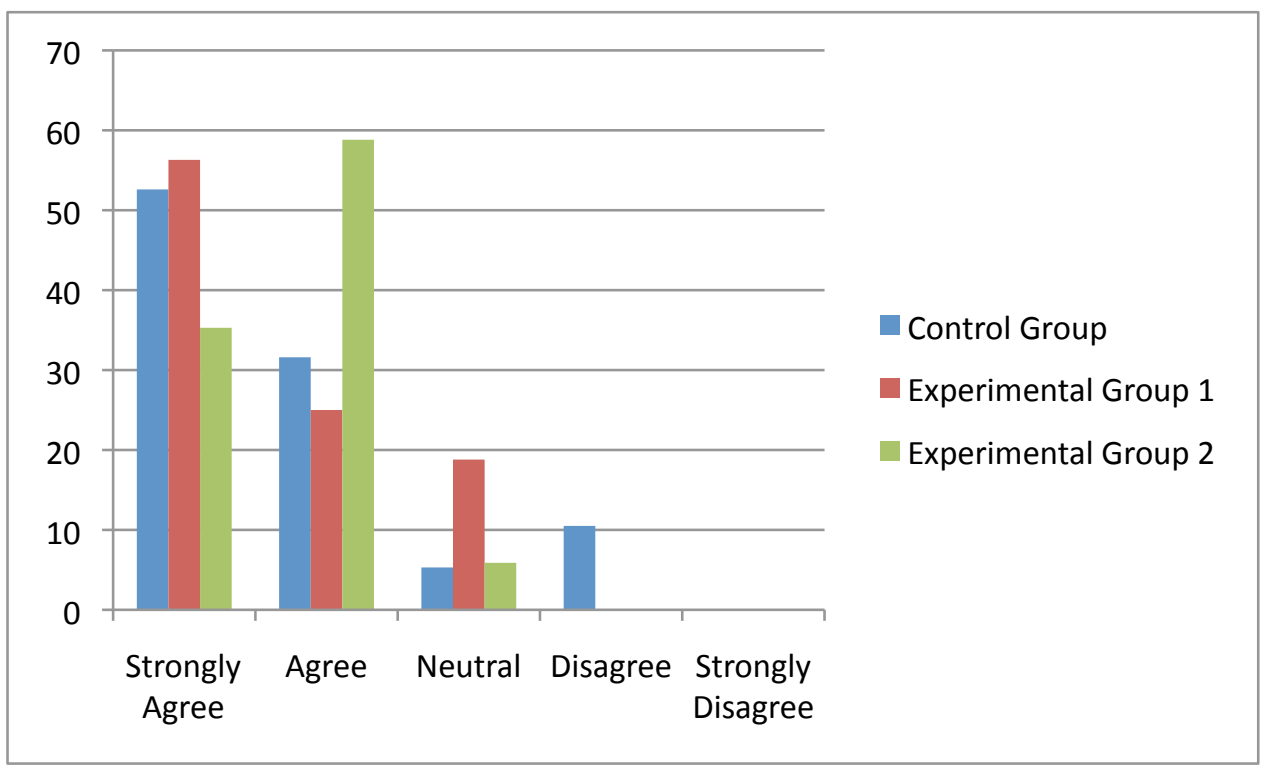

Figure 3. Student responses to the question

"I learned a great deal in this course" 


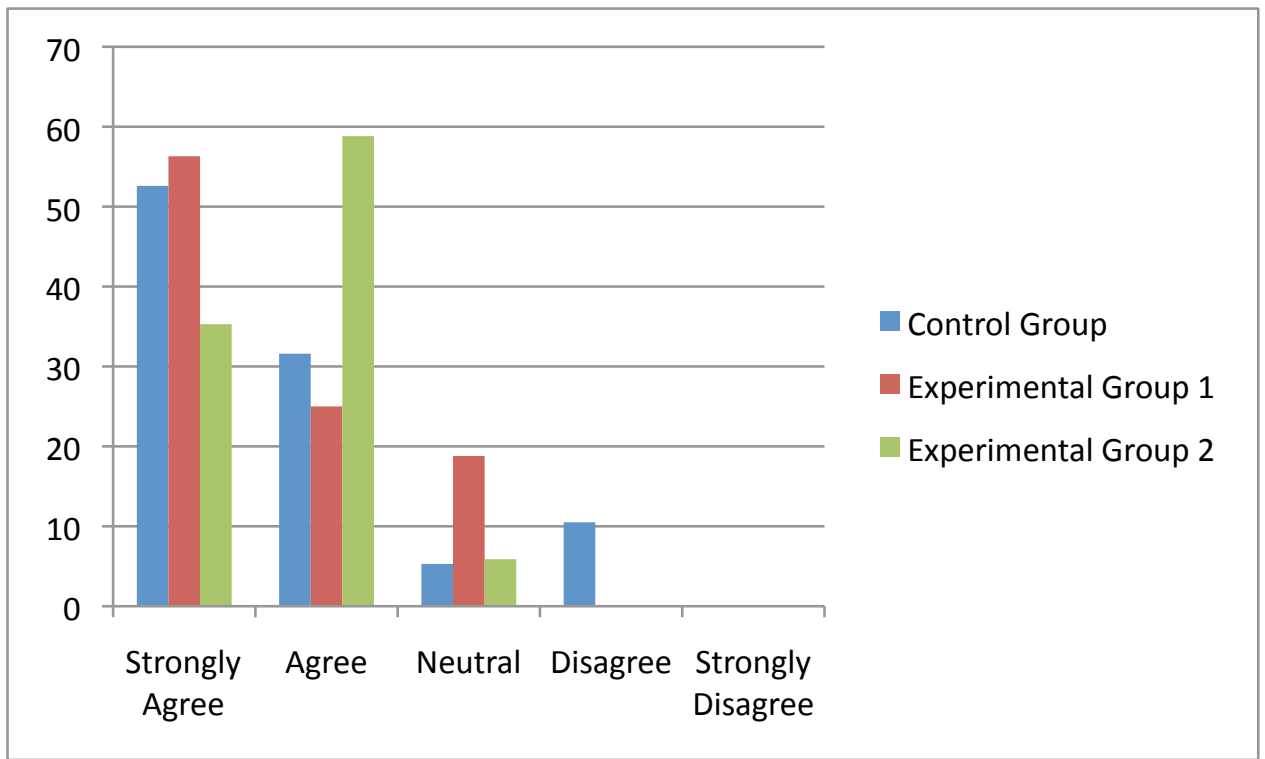

Figure 4. Student responses to the question "I participated regularly in message board discussions"

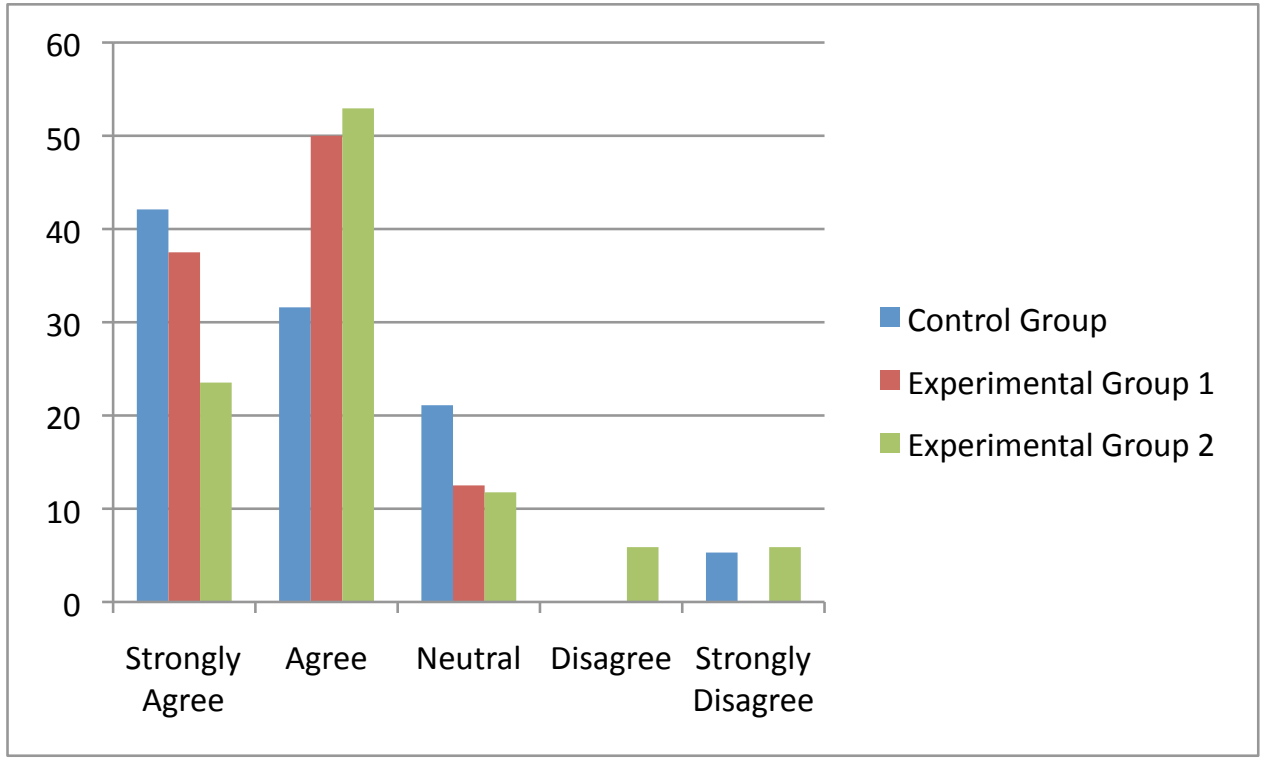

Figure 5. Student responses to the question

"The message board discussion was valuable in my learning experience" 


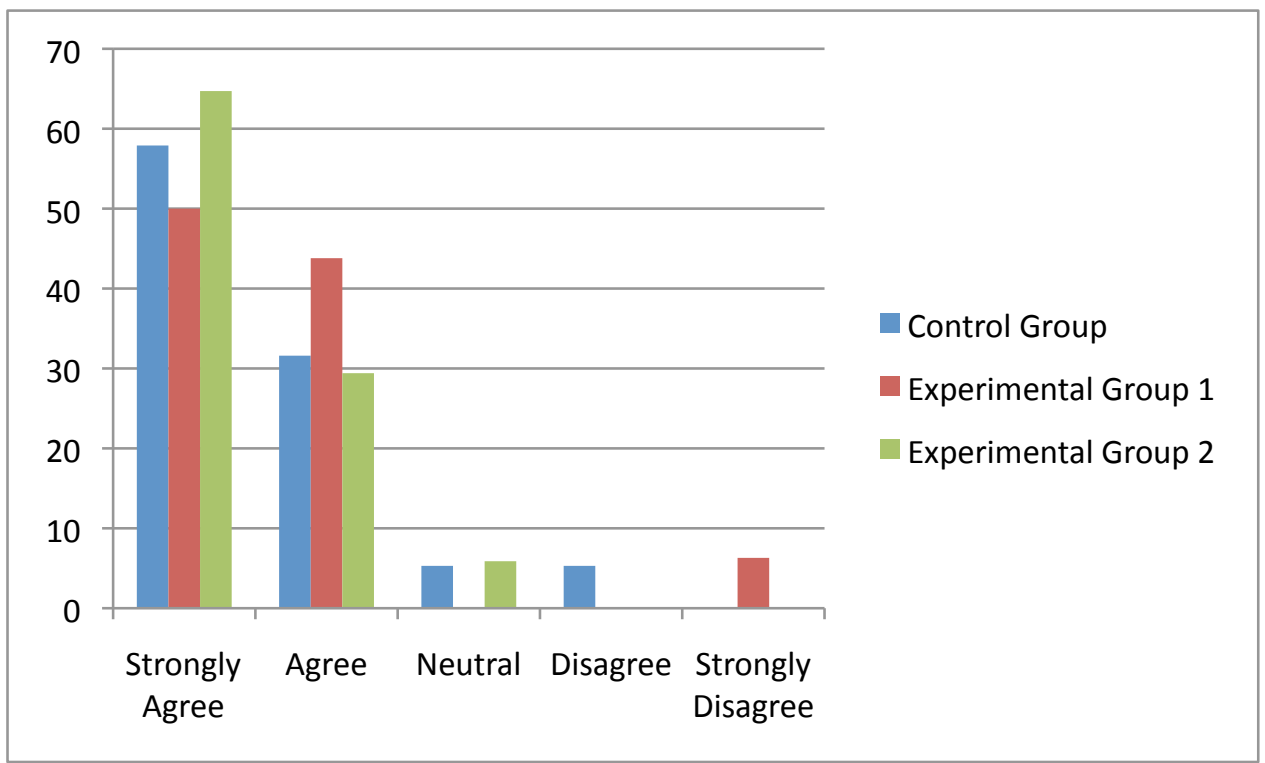

Figure 6. Student responses to the question

"The instructor for this course interacts effectively with the students"

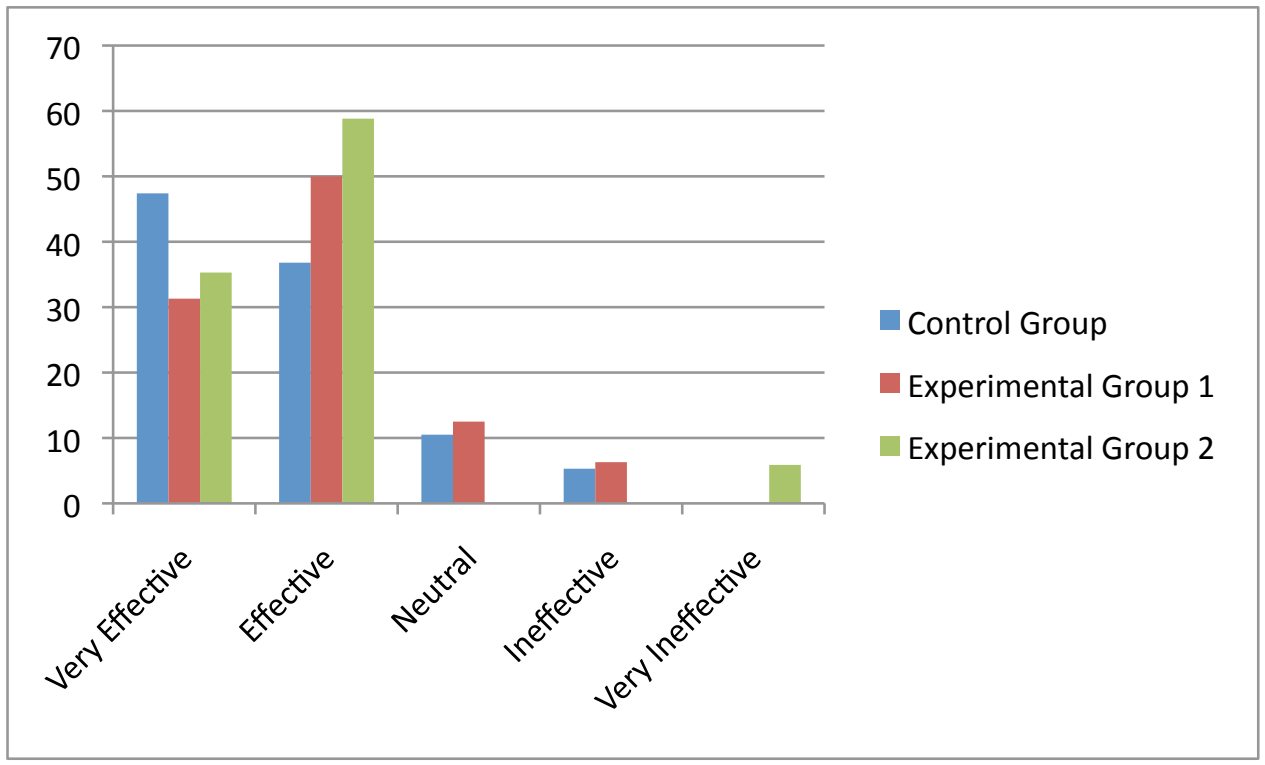

Figure 7. Student responses to the question

"Rate the overall quality of instruction in this course as it contributed to your learning (Try setting aside your feelings about the course itself)" 


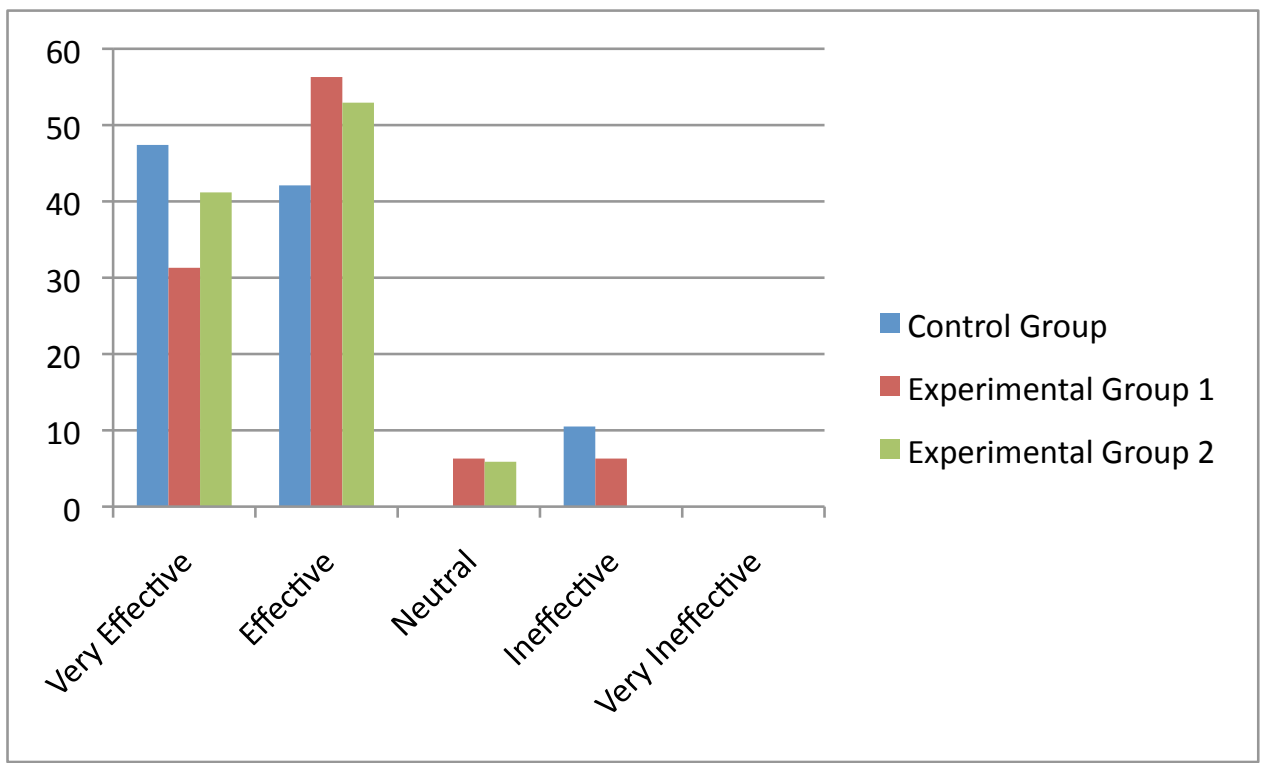

Figure 8. Student responses to the question

"Rate the overall value of this course to you as it contributed to your learning (Try to set aside your feelings about the instructor)"

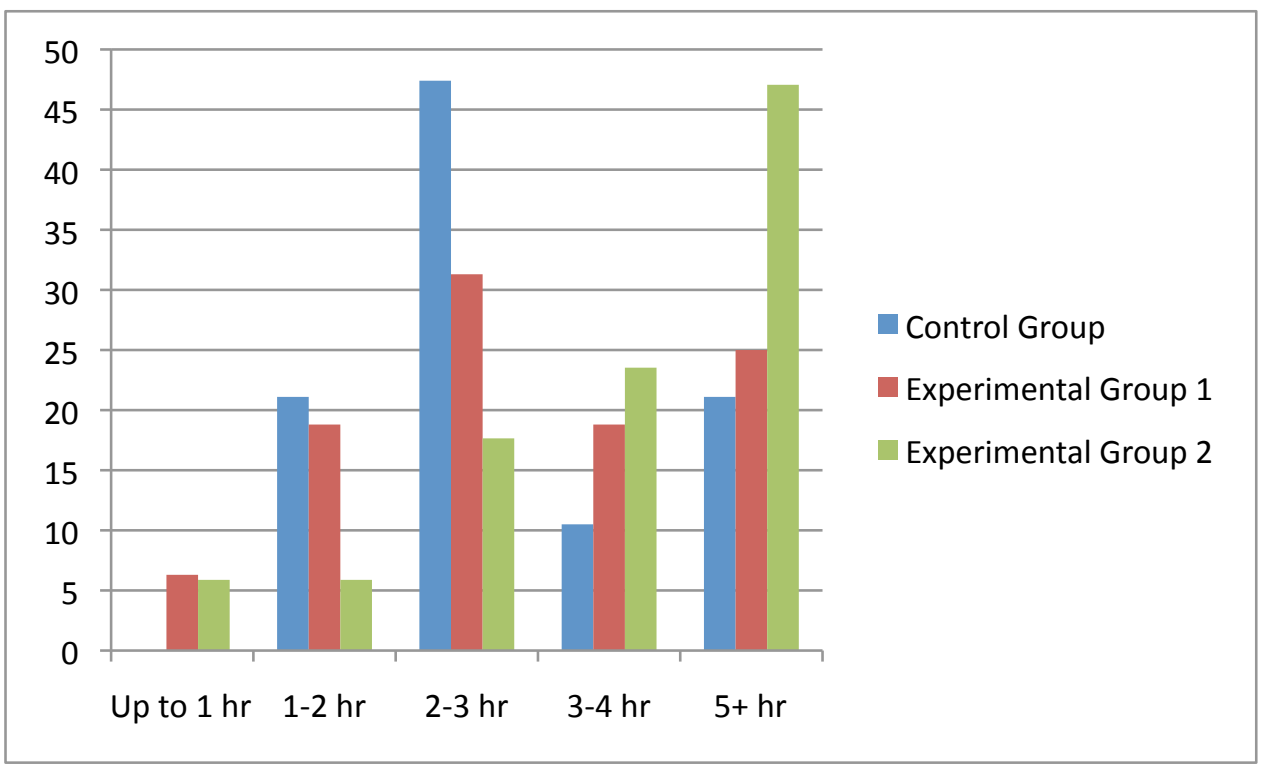

Figure 9. Student responses to the question

"How many hours per week on average do you spend on the message board?"

\section{Discussion}

\section{Statistical analysis}

The results indicate that the ratio of posts per discussion question to learners is considerably higher in the combined experimental group. The average number of responses over all questions over the semester for the combined experimental group was 3.88 while the average number of responses for the control group was 2.77. Table 1 shows that it is clear that the two groups are markedly different on all descriptive statistics except for their standard deviations. 


$\begin{array}{lllllll}\text { Variable } & N & \text { Mean } & \text { StDev } & \text { Minimum } & \text { Maximum } & \text { Median } \\ \text { Control } & 12 & 2.7708 & 0.3394 & 2.2500 & 3.5000 & 2.6750 \\ \text { Combined } & 24 & 3.8883 & 0.3613 & 3.3800 & 4.6800 & 3.8150\end{array}$

Table 1. Statistical analysis

\section{Instructor evaluations}

In reviewing the histograms presented in the results section, the experimental groups' instructor evaluations scored higher than the control group when combing percentages for the strongly agree and agree categories together, and the very effective to effective categories together. For instance, in combining the strongly agree with the agree categories, $84.2 \%$ of students in the control group found the course to be intellectually stimulating compared with $87.6 \%$ in the first experimental group and $94.12 \%$ in the second experimental group. And in combining the strongly agree with the agree categories, $73.7 \%$ of the students in the control group found the message board discussion to be valuable in their learning experience compared with $87.5 \%$ in the first experimental group and $76.47 \%$ in the second experimental group. Furthermore, the histograms show how students perceived their overall value of the course to be higher in the experimental groups than in the control group. When combining the very effective and the effective categories and using the average experimental percentage scores, students perceived both the quality of instruction and value of the course to be higher than those in the control group. The amount of time students spent on the discussion board was significantly higher in the experimental groups than in the control groups. In the control group, $31.6 \%$ of the students spent three or more hours per week posting on the discussion board. In the experimental groups, students spent $43.8 \%$ in the first experimental group and $70.59 \%$ in the second experimental group in terms of spending three or more hours on the discussion board content.

\section{Implications and recommendations}

This study is significant because it provides researched guidelines and a rubric that instructors can use to improve the quantity and quality of online discussions in project management or business class discussion forums. The research suggests that specific grading activities, such as the rubric, promote better quality discussions and a higher sense of community. Additionally, student-to-student interaction can provide online instructors with a strategy to increase student satisfaction in terms of their perceived value and quality of instruction that could aid in retention. The results suggest that the use of a rubric specifically designed to engage the student and expand the student's learning experience can increase online classroom participation and, as a consequence, enhance the educational experience of the online project management student, bolster the quality of the online program and enhance the reputation of the business school. The research outcome is important as the inclusion of effective grading rubrics for discussion forums in online project management courses represents a movement toward higher standards in the classroom, increased satisfaction and learning for the students, and improved quality and quantity of online discussions leading to an enhanced online project management program.

\section{References}

Arbaugh, J.B. 2005, Is there an optimal design for on-line MBA courses? Academy of Management Learning and Education, vol. 4, no. 2, 135-149.

Arbaugh, J.B., \& Rau, B.L. 2007, A study of disciplinary, structural, and behavioral effects on course outcomes 
in online MBA courses, Decision Sciences Journal of Innovative Education, vol. 5, no. 1, 65-95.

Berge, Z. \& Collins, M. 1995, Computer-mediated communication and the online classroom in distance learning, Computer-Mediated Communication Magazine, vol. 2, no. 4, retrieved from http://sunsite.unc.edu/cmc/mag/1995/apr/berge.html.

Blount, Y., \& McNeill, M. 2011, Fostering independent learning and engagement for postgraduate students using a publisher-supplied software program, International Journal of Educational Management, vol. 25, no. 4, 390-404, doi: 10.1108/09513541111136667.

Czaplewski, A.J. 2009, Computer-assisted grading rubrics: automating the process of providing comments and student feedback, Marketing Education Review, vol. 19, no. 1, 29-36.

Dawson, S., Burnett, B. \& O’Donohue, M. 2006, Learning communities: an untapped sustainable competitive advantage for higher education, International Journal of Educational Management, vol. 20, no. 2, 127 139, doi: 10.1108/09513540610646118.

Elgort, I. 2011, Dealing with complexity through course design, On the Horizon, vol. 19, no. 2, 97-108, doi: $10.1108 / 10748121111138290$.

Fauske, J. \& Wade, S. E. 2004, Research on practice online: conditions that foster democracy, community, and critical thinking in computer-mediated discussions, Journal of Research on Technology in Education, vol. 36 , no. $2,137-153$.

Gilbert, P.K. \& Dabbagh, N. 2005, How to structure online discussions for meaningful discourse: a case study, British Journal of Educational Technology, vol. 36, no. 1, 5-18.

Goodrich Andrade, H. 2005, Winter, Teaching with rubrics: the good, the bad, and the ugly, College Teaching, vol. 53, 27-30, doi: 761703241.

Goodrich, H. 1996, Understanding rubrics. Educational Leadership, vol. 54, no. 4, 14-17. doi: 10533927

Kay, R.H. 2006, Developing a comprehensive metric for assessing discussion board effectiveness, British Journal of Educational Technology, vol. 37, no. 5, 761-783, doi: 1074400071.

Langan, T. 1997, Online education: a student's perspective, Campus-Wide InformationSystems, vol. 14, no. 4, 128-132.

Li, J. 2011, Quality of work life and career development: perceptions of part-time MBA students, Employee Relations, vol. 33, no. 3, 201-220, doi: 10.1108/01425451111121740.

Menkhoff, T., Chian, L.K., Yian, T.T., Wah, C.Y.\& Wong, Y.K. 2011, Engaging knowledge management learners through web-based ICT: an empirical study, The Journal of Information and Knowledge Management Systems, vol. 14, no. 2, 132-151, doi: 10.1108/03055721111134781.

Owston, R. 1997, The World Wide Web: a technology to enhance teaching and learning? Educational Researcher, vol. 26, no. 2, retrieved from http://www.edu.yorku.ca/ rowston/article.html.

Popham, W.J. 1997, October, What's wrong — and what's right — with rubrics, Educational Leadership, vol. 55, 72-75, doi: 17239567.

Rovai, A. 2003, Strategies for grading online discussions: Effects on discussions and classroom community in internet-based university courses. Journal of Computing in Education, vol. 15, no. 1, 89-107, doi: 2164863731.

Strang, K. D. 2011, How can discussion forum questions be effective in online MBA courses? Campus-Wide Information Systems, vol. 28, no. 2, 80-92, doi: 10.1108/10650741111117789.

Swan, K. 2001, Virtual interaction: design factors affecting student satisfaction and perceived learning in asynchronous online courses. Distance Education, vol. 22, no. 2, 306-331, doi: 98666711.

Weltzer-Ward, L. 2011, Content analysis coding schemes for online asynchronous discussion, Campus-Wide Information Systems, vol. 28, no. 1, 56-74, doi: 10.1108/10650741111097296.

\section{About the authors:}

Dr Margaret R. Lee, $P M P \AA$, has a doctorate in Organization and Management/Project Management and is principal for LEE Consultants. Lee's consulting practice assists educational facilities in strategically planning virtual project management and e-leadership development curricula and also provides basic project management training and consultation for international corporations. Her professional experience includes corporate trainer, instructional designer and project manager. She currently teaches at Blackburn College in Carlinville, Illinois, and online for Florida Institute of Technology and the University of Northwestern Ohio. Lee is a referee for the International Journal of Project Management, on the Editorial Review Board for the International Journal of Mentoring and Coaching in Education, and a reviewer for the 2012 Project Management Institute's Research \& Education Conference Committee. An active member of the PMI Central Illinois Chapter, she is a facilitator for their Springfield, Illinois, division's PMP Exam Review Course Certification Training.

Email: peg.lee1@gmail.com 
Dr Deborah S. Carstens PMP®, joined Florida Tech in fall 2003 where she instructs both on campus and online courses. She is an Associate Professor in Information Systems and the Academic Chair for the Project Management Track in the Online MBA Program. Previously, she worked for NASA Kennedy Space Center (KSC) from 1992 through 2003. In her career both at Florida Tech and NASA, she has managed over a million dollars of funded research. She instructs undergraduate and graduate courses in MIS, human-computer interaction and project management. She has had an opportunity to work on funded research grants from the Small Business Administration, Florida Department of Education, Department of Health \& Human Services, NASA and other locally funded studies in task analysis, human error, patient safety, usability, learning effectiveness and government accountability. She is currently working on a project management tools and techniques textbook being published in 2013.

Email: carstens@fit.edu

Dr Linda C. Malone is a Professor Emeritus in Industrial Engineering from the University of Central Florida. She got her PhD degree in statistics from Virginia Tech after having gotten her BS and MS degrees in mathematics (at Emory and Henry College and University of Tennessee respectively). Her primary research interests include Response Surface Analysis and Quality. Malone has published 94 refereed articles, supervised $26 \mathrm{PhD}$ student dissertations and authored one statistics textbook. She was an associate editor of the Journal of Statistical Computation and Simulation for over 25 years and was a founding co-editor of the STATS Magazine. She was awarded the honor of Fellow of the American Statistical Association.

Email: Linda.malone@ucf.edu 
Appendix A: Discussion forum rubric for the two experimental groups

\begin{tabular}{|c|c|c|c|c|c|c|}
\hline WEEK \# & DQ $1=25 \%$ & & & & & \\
\hline & $\begin{array}{l}\text { Insight/ } \\
\text { Depth }\end{array}$ & Book ref? & Other ref? & $\begin{array}{c}\text { Answer } \\
\text { all/Complete }\end{array}$ & $\begin{array}{c}\text { Example/ } \\
\text { Experience? }\end{array}$ & $\begin{array}{c}\text { Possible } \\
\text { Total } \\
\end{array}$ \\
\hline (points) & 5 & 5 & 5 & 5 & 5 & 25 \\
\hline $\begin{array}{l}\text { STUDENT } \\
\text { NAME }\end{array}$ & 0 & 0 & 0 & 0 & 0 & 0 \\
\hline WEEK \# & $\begin{array}{l}\text { Response } \\
\text { DQ } 1=25 \%\end{array}$ & & & & & \\
\hline & $\begin{array}{c}\text { Insight/ } \\
\text { Depth }\end{array}$ & Book ref? & Other ref? & $\begin{array}{c}\text { Responses } \\
2 \\
\end{array}$ & $\begin{array}{c}\text { Responses } \\
>2\end{array}$ & $\begin{array}{c}\text { Possible } \\
\text { Total } \\
\end{array}$ \\
\hline (points) & 5 & 5 & 5 & 5 & 5 & 25 \\
\hline $\begin{array}{l}\text { STUDENT } \\
\text { NAME }\end{array}$ & 0 & 0 & 0 & 0 & 0 & 0 \\
\hline WEEK \# & DQ $2=25 \%$ & & & & & \\
\hline & $\begin{array}{l}\text { Insight/ } \\
\text { Depth }\end{array}$ & Book ref? & Other ref? & $\begin{array}{c}\text { Answer } \\
\text { all/Complete }\end{array}$ & $\begin{array}{c}\text { Example/ } \\
\text { Experience? }\end{array}$ & $\begin{array}{c}\text { Possible } \\
\text { Total }\end{array}$ \\
\hline (points) & 5 & 5 & 5 & 5 & 5 & 25 \\
\hline $\begin{array}{l}\text { STUDENT } \\
\text { NAME }\end{array}$ & 0 & 0 & 0 & 0 & 0 & 0 \\
\hline WEEK \# & $\begin{array}{l}\text { Response } \\
\text { DQ2=25\% }\end{array}$ & & & & & \\
\hline & $\begin{array}{c}\text { Insight/ } \\
\text { Depth }\end{array}$ & Book ref? & Other ref? & $\begin{array}{c}\text { Responses } \\
2 \\
\end{array}$ & $\begin{array}{c}\text { Responses } \\
>2\end{array}$ & $\begin{array}{c}\text { Possible } \\
\text { Total }\end{array}$ \\
\hline (points) & 5 & 5 & 5 & 5 & 5 & 25 \\
\hline $\begin{array}{l}\text { STUDENT } \\
\text { NAME }\end{array}$ & 0 & 0 & 0 & 0 & 0 & 0 \\
\hline WEEK \# & TOTAL & & & & & \\
\hline $\begin{array}{l}\text { STUDENT } \\
\text { NAME }\end{array}$ & 0 & & & & & \\
\hline
\end{tabular}




\section{Appendix B: Instructional post for the two experimental groups, "Grading for Posts and Responses"}

What makes an excellent post? For your convenience, I have copied the following from the Expectations post in our Main Discussion Board that you were encouraged to read and sign off on in our Class News.

"Discussion Board Questions (DQ) Posts and Responses:

Your grade is broken down into four parts: 25\% DQ\#1 post, 25\% DQ\#1 responses, 25\% DQ\#2 post, 25\% DQ\#2 responses. Your grade for each part is determined by the following criteria.

Discussion Board posts and responses should be graduate school level writing, using APA formatting for references. All posts and responses should be generally free of errors. It is suggested that you write your posts in Microsoft Word and use spell check and grammar check prior to submitting them.

Discussion posts should be insightful, thorough and interesting. They are insightful through your analysis of the material and thorough in your complete response to the question. An excellent discussion post demonstrates that you understand the topic and, if possible, shares personal examples and experiences on the subject. Your post should be interesting by including new and useful information for the readers. The excellent post uses two or more examples from the textbook or other resources (journal articles, books, white papers, professional/academic web sites, etc.). Everyone in the class has the textbook, so bringing new knowledge and new references to the discussion distinguishes the higher-level posting.

Your responses to other students should demonstrate substantive analysis and/or evaluation of their discussion post. A response should be more than agreement and be an in-depth evaluation or discussion of the post. Providing new resources that relate to the response can do this. Again, everyone has already read the text; so providing information from the text is expected for a good response. An excellent response includes personal stories, new resources, examples or experiences that relate to the topic, or asks questions regarding the response post to encourage conversation. Providing a contrasting view and developing your case for it using academic references is an example of an excellent response post.

Another example of excellence in the discussion classroom is stepping forward to answer another student's question(s) on a topic or leading that learner to a resource that will help answer his or her question(s). In addition, those who go beyond just doing the required number of responses to other students' postings show that their abilities are above average."

\section{Here is some additional information on how the $\mathbf{2 5 \%}$ for each part is broken down even further in the grading rubric. (Also, see attached sample student rubric.)}

25\% DQ\#1 post, 25\% DQ\#1 responses, 25\% DQ\#2 post, 25\% DQ\#2 responses.

Discussion posts should be insightful, thorough and interesting. They are insightful through your analysis of the material (5\%) and thorough in your complete response (5\%) to the question. An excellent discussion post demonstrates that you understand the topic and, if possible, shares personal examples and experiences on the subject (5\%). Your post should be interesting by including new and useful information for the readers. The excellent post uses two or more examples from the textbook (5\%) and other resources (journal articles, books, white papers, professional/academic web sites, etc.) (5\%). Everyone in the class has the textbook, and bringing new knowledge and new references to the discussion distinguishes the higher-level posting.

Your responses to other students should demonstrate substantive analysis and/or evaluation of their discussion post. A response should be more than agreement and be an in-depth evaluation or discussion of the post (5\%). Two responses to other learners are required for each discussion question (5\%). (You are encouraged to continue conversations with your professor, but your responses to the professor will not count as responses to other students.) An excellent response includes personal stories, new resources, examples or experiences that relate to the topic, or asks questions regarding the response post to encourage conversation. Providing a contrasting view and developing your case for it using academic references is an example of an excellent response post. Providing new resources that relate to the response can do this (5\%). Again, everyone has the text $(5 \%)$; so providing information from the text is expected for a good response.

Another example of excellence in the discussion classroom is stepping forward to answer another student's question(s) on a topic or leading that learner to a resource that will help answer his or her question(s). In addition, those who go beyond just doing the required number of responses to other students' postings show that 
their abilities are above average (5\%).

If you respond to more than the required minimum of two other learners, your additional posts (response posts \#3, 4, 5, etc.) do not have to cite Portny or an outside source. You should, of course, substantiate your thoughts, so citations are encouraged. However, just normal conversation and sharing of ideas, tips and experiences is also acceptable for posts beyond the required minimum of two other learners. Two of your response postings are required to reference Portny and an outside source (see the grading rubric). Our goal in BUS 5661 is to learn from each other and our discussion forums are the way we can do this!

Please let me know if you have any questions. We are working toward excellence in our postings in BUS 5661 and the sharing of information/resources/references is vital to the project management profession.

YOU WILL NEED TO CONFIRM THAT YOU HAVE READ THIS DOCUMENT BY REPLYING TO THIS POSTING IN THE MESSAGE BOARD.

Thank you. 


\section{Appendix C: Instructor Evaluation Comments on the Discussion Board from Students for the Control Group and Experimental Groups}

\section{Control Group Discussion Board Related Comments:}

"If these (discussion board questions) are going to count for $40 \%$ of the students overall grade, they really need to be much more effective and lead to good constructive dialogue. Then the instructor needs to lead the discussion down other paths from there. When 20 people are answering the same exact question and when there really isn't that much to be said on the subject or it was already clearly explained in the textbook, then there isn't much value added!"

"Instructor gave excellent feedback on my assignments (discussion board questions) and it helped me get a better grade in later assignments."

\section{Experimental Group Discussion Board Related Comments:}

"As a PM I think that the harsh discussion requirements are right in tune with reality and that the difficulties are a learning opportunity in and of themselves. When you are managing a project and disagree with the customer on a requirement (that you agreed upon by signing), you are rarely going to see the results you expect.

Especially if nothing has been said right up until the time it became difficult for you to execute. The discussion requirements are difficult to meet without question; they are not, however, impossible.

Change management is a part of PM work but the changes are usually done on the project side and not demanded of the customer's requirements. Let's look at our taking this course as if we were project managers.

- Project initiation stage - We sign up for the course and the instructor agrees to teach the course.

- Project planning and design stage - We prepare for the course and the instructor outlines the syllabus.

- Project execution and construction stage - This would be week one. You can still get a return on your investment if you decide the course is not for you. You have a chance to review the course materials (requirements) and to question them. By saying nothing, signing the statement of understanding and remaining in the course you have agreed to the project terms.

- Project monitoring and controlling systems - We continue with the course work

- Project completion - Final Exam and hopefully, the taking from this course a wealth of knowledge that will prove useful in your career."

"Your stress on providing scholarly citations is something of a big take away for me (and also to not speed-read important stuff."). I was forced to do some extra reading for the DQs thanks to your strictness."

"I learned a lot in this class that will help me in my career."

"I have enjoyed this class, the material and you as the instructor more than any other I have had while pursuing my MBA ..."

"Thank you for a great term, I learned a great deal and this should give me a good foundation for the rest of my project management courses."

"I am very excited to implement the knowledge I have gained from this course into my current career as a ..."

"This class by far has been the most helpful in my understanding of project management."

"Thank you for taking the time to explain the standard for supporting references on the MBA level. I am enjoying the class while getting insight from others in the Project Management field." 